\title{
Further observations on the mean velocity distribution in fully developed pipe flow
}

\author{
By B. J. MCKEON, J. LI, $\dagger$ W. JIA N G $\ddagger$, \\ J. F. MORRISONף AND A. J. SMITS \\ Department of Mechanical and Aerospace Engineering, Princeton University, \\ Princeton, NJ 08544-0710, USA
}

(Received 7 October 2002 and in revised form 19 September 2003)

The measurements by Zagarola \& Smits (1998) of mean velocity profiles in fully developed turbulent pipe flow are repeated using a smaller Pitot probe to reduce the uncertainties due to velocity gradient corrections. A new static pressure correction (McKeon \& Smits 2002) is used in analysing all data and leads to significant differences from the Zagarola \& Smits conclusions. The results confirm the presence of a power-law region near the wall and, for Reynolds numbers greater than $230 \times 10^{3}$ $\left(R^{+}>5 \times 10^{3}\right)$, a logarithmic region further out, but the limits of these regions and some of the constants differ from those reported by Zagarola \& Smits. In particular, the log law is found for $600<y^{+}<0.12 R^{+}$(instead of $600<y^{+}<0.07 R^{+}$), and the von Kármán constant $\kappa$, the additive constant $B$ for the $\log$ law using inner flow scaling, and the additive constant $B^{*}$ for the log law using outer scaling are found to be $0.421 \pm 0.002,5.60 \pm 0.08$ and $1.20 \pm 0.10$, respectively, with $95 \%$ confidence level (compared with $0.436 \pm 0.002,6.15 \pm 0.08$, and $1.51 \pm 0.03$ found by Zagarola \& Smits). The data also confirm that the pipe flow data for $\operatorname{Re}_{D} \leqslant 13.6 \times 10^{6}$ (as a minimum) are not affected by surface roughness.

\section{Introduction}

\subsection{Background and previous work}

Zagarola \& Smits (1998) (referred to hereinafter as ZS) presented measurements in fully developed pipe flow for Reynolds numbers in the range $31 \times 10^{3}$ to $35 \times 10^{6}$ to study the scaling of the mean velocity profile. They found two overlap regions: a power law for $60<y^{+}<500$, and, for Reynolds numbers greater than $400 \times 10^{3}$, a log law in the region $600<y^{+}<0.07 R^{+}$. Here, $y^{+}=y u_{\tau} / v, y$ is the distance from the wall, $u_{\tau}=\sqrt{\tau_{w} / \rho}, v$ is the kinematic viscosity, $\tau_{w}$ is the shear stress at the wall, and $\rho$ is the fluid density. Also, $R^{+}=R u_{\tau} / v$ where $R$ is the radius of the pipe $(=D / 2)$. These findings were supported by a new scaling argument based on dimensional analysis. For high Reynolds numbers, the scaling for the mean velocity profile in fully developed turbulent pipe flow may be expressed in terms of an inner-layer scaling given by

$$
U=f^{\prime}\left(y, u_{i}, v, R\right)
$$

$\dagger$ Permanent address: School of the Built Environment, Victoria University of Technology, PO Box 14428, MCMC, Melbourne, Australia.

$\ddagger$ Permanent address: CARDC, PO Box 211 Mianyang, Sichuan 621000, P. R. China.

If Permanent address: Department of Aeronautics, Imperial College, London SW7 2BY, UK. 
and an outer-layer scaling given by

$$
U_{c}-U=g^{\prime}\left(y, u_{0}, v, R\right)
$$

where $U$ is the mean velocity, $U_{c}$ is the centreline velocity, and $f^{\prime}$ and $g^{\prime}$ denote a functional dependence. The inner velocity scale $u_{i}$ is always taken to be $u_{\tau}$, but choosing the outer velocity scale $u_{0}$ is more controversial, as will be seen below. Non-dimensionalizing equations (1.1) and (1.2) gives, respectively,

$$
\begin{gathered}
U^{+}=f\left(y^{+}, R^{+}\right), \\
\frac{U_{c}-U}{u_{0}}=g\left(\eta, R^{+}\right),
\end{gathered}
$$

where $U^{+}=U / u_{\tau}$ and $\eta=y / R$. ZS showed that the velocity scaling in the overlap region (where $y^{+} \gg 1$ and $\eta \ll 1$ ) can be of two types: complete similarity where $u_{0} / u_{\tau}$ is independent of Reynolds number, and incomplete similarity where $u_{0} / u_{\tau}$ continues to depend on Reynolds number. In the case of complete similarity, matching of the velocity gradients in the overlap region leads to a logarithmic velocity profile, which can be expressed in inner-layer variables as

$$
U^{+}=\frac{1}{\kappa} \ln y^{+}+B
$$

and in outer-layer variables as

$$
\frac{U_{c}-U}{u_{\tau}}=-\frac{1}{\kappa} \ln \eta+B^{*}
$$

where $\kappa$ (usually called the von Kármán constant), $B$ and $B^{*}$ are constants independent of Reynolds number.

Alternatively, in the case of incomplete similarity, matching the velocities as well as the velocity gradients in the overlap region yields a power-law dependence which in terms of inner-layer variables may be written as

$$
U^{+}=C y^{+\gamma}
$$

where the coefficient $C$ and the exponent $\gamma$ are independent of Reynolds number. The study by ZS showed that the power-law constants were given by $C=8.70$ and $\gamma=0.137$, and the log-law constants were given by $\kappa=0.436 \pm 0.002, B=6.15 \pm 0.08$, and $B^{*}=1.51 \pm 0.03$. These values of the log-law constants are different from the commonly accepted values of about $0.41,5.0$ and 0.8 , respectively. for the so-called 'standard log law' discussed below.

ZS also proposed a new velocity scale for the outer region, $u_{0}=U_{c}-\bar{U}$. They argued that $u_{0}=U_{c}-\bar{U}$ was a more representative outer velocity scale than the friction velocity, which describes the inner flow and is impressed upon the outer region. Since the ratio $\left(U_{c}-\bar{U}\right) / u_{\tau}$ becomes constant at high Reynolds number, there is no change to the overlap analysis leading to a log law at these Reynolds numbers. At low Reynolds numbers (high enough that an overlap region still exists), the variation of $\left(U_{c}-\bar{U}\right) / u_{\tau}$ with Reynolds number means that matching velocity gradients does not lead to a Reynolds-number-independent condition. However matching velocities and velocity gradients leads to the power law of equation (1.7).

ZS obtained their measurements using a round Pitot probe of $0.9 \mathrm{~mm}$ OD. A number of corrections were made to the data, including corrections to the Pitot tube pressure for the effects of turbulence, viscosity, and velocity gradient, and corrections to the static pressure for viscous effects. Subsequent examination of the ZS data 


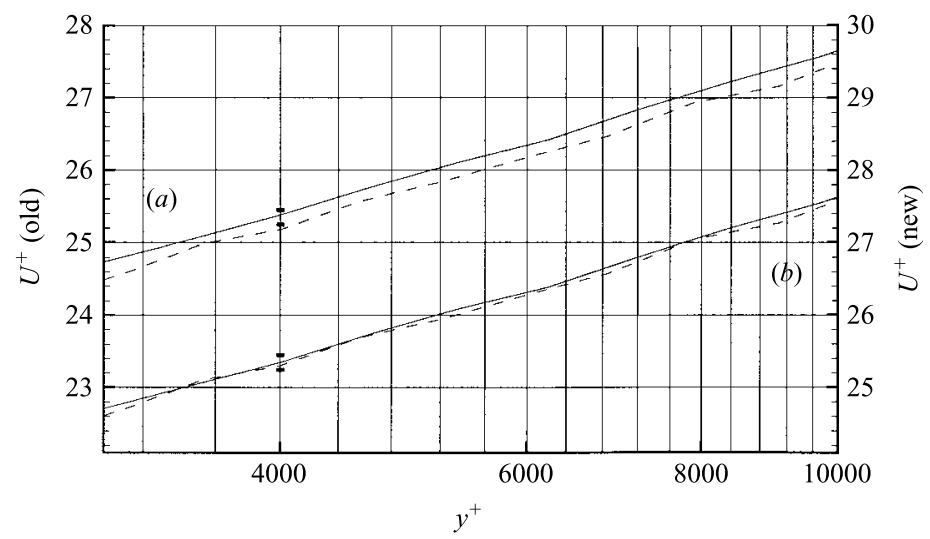

FIGURE 1. Velocity profiles for the $0.9 \mathrm{~mm}(\mathrm{ZS})$ data at $R e_{D}=3 \times 10^{6}$ (solid lines) and $10 \times 10^{6}$ (dashed lines): (a) results using Shaw's (1960) correction as reported by ZS; $(b)$ results using correction according to McKeon \& Smits (2002).

revealed slight deviations between the velocity profiles in inner scaling (figure $1 a$ ). The deviations (also called 'steps') appear as departures from the logarithmic region for $\operatorname{Re}_{D}>3 \times 10^{6}$ and increase with Reynolds number.

In $\mathrm{ZS}$, the comparison of the data with the log law and the power law was done by fitting the scaling laws to the mean velocity profile. As pointed out by W. George (private communication), the mean velocity itself is not a very sensitive quantity in distinguishing a log law from a power law because the differences are small. This is especially true at low Reynolds number. Fractional differences were therefore used, as suggested by Zagarola, Perry \& Smits (1997). The fractional difference $E=\Delta U^{+} / U^{+}$ between a fitted curve and the measured data is defined as

$$
E=\frac{\Delta U^{+}}{U^{+}}=1-\frac{U_{\text {fit }}^{+}}{U_{\text {measured }}^{+}} \text {. }
$$

Zagarola et al. suggested that a good fit to the experimental data should result in random relative errors that are less than the mean experimental error.

Traditionally, dimensional analysis using $u_{0}=u_{\tau}$ has yielded a log law (Millikan 1938). The so-called 'standard log law' arose from the conclusions of several studies in low-Reynolds-number boundary layers, including that by Bradshaw (1976), that the overlap region was best represented by a $\log$ law with $\kappa \approx 0.41$ and $B \approx 5.0$ (Schlichting 1979).

Österlund et al. (2000) in a more recent study of boundary layers found a log law with $\kappa=0.38$ and $B=4.1$ in the range $200 / \delta^{+}<y / \delta<0.15$ for $R e_{\theta}>6000$. Although this log law appears over a rather short region in $y^{+}$(200 to about 1000), we should note that this includes virtually all boundary layer studies, where few studies have a large enough Reynolds number range to investigate the overlap region with an upper extent where $y^{+} \geqslant 1000$. This includes the work by Österlund et al. where the maximum value of $R e_{\theta}$ was 27000 .

In a medium-Reynolds-number channel flow $\left(R e_{h}<1.2 \times 10^{5}\right.$ based on half-channel height and mean velocity, $1000<R e_{\tau}<5000$ ), Zanoun et al. (2002) found a similar value of the von Kármán constant, $\kappa=0.379$, with $B=4.05$, for $R e_{\tau}>2000$ within the limits $y^{+}>100$ and $y / h<0.15$. However, they also suggested that a log law may 
be valid for limits reaching $y^{+}=50$ and $y / h=0.9$, which presumably yields different log-law constants.

\subsection{Corrections to the measurements}

Perry, Hafez \& Chong (2001) attributed the steps in the data (which were exacerbated in their analysis by a different choice of $\kappa$ and $B$ ) to roughness effects combined with inappropriate Pitot tube corrections. Recent experiments at Princeton, however, have demonstrated that these deviations were in fact due to inaccurate static pressure corrections (McKeon \& Smits 2002) and the absence of a wall term in the Pitot correction. ZS used the static pressure correction proposed by Shaw (1960), who suggested that the error, $\Delta p$, depends only on $d_{t}^{+}$and reaches an asymptotic value of approximately $3.0 \tau_{w}$ for $d_{t}^{+} \geqslant 1000$, where $d_{t}^{+}=u_{\tau} d_{t} / v$ and $d_{t}$ is the hole diameter. McKeon \& Smits (2002) have shown instead that the error continues to grow with increasing Reynolds number as long as the ratio $d_{t} / D$ is small, and reaches a value of greater than $7 \tau_{w}$ at the highest Reynolds numbers obtained in the pipe, where $d_{t}^{+} \approx 6500$. Although the new correction corresponds to a change of less than $1 \%$ in the maximum dynamic pressure for all cases presented here, the new static pressure correction removes the deviations observed in the ZS profiles to within the limits of experimental uncertainty (see figure $1 b$ ).

To understand the effect of Pitot probe corrections on the similarity scaling and the value of the log-law constants, a further study was undertaken by McKeon et al. (2003) using four different sized Pitot tubes, measuring $0.3 \mathrm{~mm}$ to $1.83 \mathrm{~mm}$ OD. A new correction scheme was suggested that collapses the data markedly better than other wall corrections methods, including that suggested by MacMillan (1956), for $y<2 d$, where $d$ is the outer diameter of the Pitot tube, and that agrees well with MacMillan's and Chue's (1975) methods over the entire Reynolds number range for $y \geqslant 2 d$.

\subsection{Current study}

ZS measured 28 velocity profiles over a range of Reynolds numbers from $31 \times 10^{3}$ to $36 \times 10^{6}$ using a $0.9 \mathrm{~mm}$ Pitot probe. We use these data, in addition to new measurements encompassing 21 profiles obtained using a Pitot probe of $0.3 \mathrm{~mm}$ replicating the ZS Reynolds numbers over the range from $74 \times 10^{3}$ to $35 \times 10^{6}$. Both sets of data included measurements of the pressure drop to determine the friction factor. The Reynolds numbers and friction factors for the mean velocity measurements analysed in this work are shown in table 1, along with the symbols that will be used in the figures that follow.

Since an appropriate Pitot correction must surely collapse data from probes of all diameters, the pipe flow mean velocity measurements were analysed in two different ways to ensure the conclusions were not affected by the Pitot correction. First, all points taken within $2 d$ of the wall, that is, where the Pitot probe corrections disagreed, were removed from the data set. The remaining data points were therefore independent of the particular Pitot tube corrections used. To be consistent with ZS the Chue (1975) correction was applied to the data, although any of the other corrections, such as MacMillan (1956), give the same results. This data set $(y \geqslant 2 d)$ was used in the analysis of the log-law shown here. Second, to investigate the near-wall behaviour, all the data points for $y \geqslant d$ were used. In this case, the data were corrected using the method suggested by McKeon et al. (2003), which gives good agreement between data from probes of different diameters for $y \geqslant d$. It should be noted that analysis of 


\begin{tabular}{|c|c|c|c|c|c|}
\hline Symbol & $R e_{D}$ & $\lambda$ & Symbol & $R e_{D}$ & $\lambda$ \\
\hline$\triangle$ & $75 \times 10^{3}$ & 0.0193 & $\boldsymbol{\nabla}$ & $2.3 \times 10^{6}$ & 0.0103 \\
\hline$\square$ & $150 \times 10^{3}$ & 0.0167 & $\star$ & $3.1 \times 10^{6}$ & 0.0099 \\
\hline$\Delta$ & $230 \times 10^{3}$ & 0.0153 & $\triangleleft$ & $4.4 \times 10^{6}$ & 0.0094 \\
\hline [ & $310 \times 10^{3}$ & 0.0147 & 1 & $6.1 \times 10^{6}$ & 0.0090 \\
\hline$\diamond$ & $410 \times 10^{3}$ & 0.0138 & 4 & $7.7 \times 10^{6}$ & 0.0086 \\
\hline ○ & $540 \times 10^{3}$ & 0.0132 & $\mathrm{P}$ & $10.2 \times 10^{6}$ & 0.0082 \\
\hline$\diamond$ & $750 \times 10^{3}$ & 0.0125 & $\triangleright$ & $13.6 \times 10^{6}$ & 0.0080 \\
\hline$\bullet$ & $1.0 \times 10^{6}$ & 0.0118 & 0 & $18.2 \times 10^{6}$ & 0.0077 \\
\hline$\nabla$ & $1.3 \times 10^{6}$ & 0.0113 & 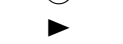 & $35.3 \times 10^{6}$ & 0.0071 \\
\hline $\mathrm{X}$ & $1.7 \times 10^{6}$ & 0.0108 & & & \\
\hline
\end{tabular}

this second data set confirmed all the conclusions derived using the first data set (the set that was independent of the Pitot tube correction used).

\subsection{Experimental considerations}

In other respects, the experimental apparatus and techniques used for the new dataset were virtually the same as those used by ZS, and their error analysis applies almost unchanged to the measurements reported here. The maximum error in velocity measurement remained $0.35 \%$. However, to improve the accuracy of the wall distance measurement, a linear encoder with an accuracy of $5 \mu \mathrm{m}$ per count was used to determine the wall distance ( $\mathrm{ZS}$ reported an accuracy of $25 \mu \mathrm{m}$ ). In addition, the starting position for the Pitot probe was determined by detecting the electrical contact between the Pitot probe and the pipe surface to an accuracy of $5 \mu \mathrm{m}$, compared to $50 \mu \mathrm{m}$ for $\mathrm{ZS}$. The accumulated position error of running the present traverse system forward and backward once over a distance of $71 \mathrm{~mm}$ was generally less than $30 \mu \mathrm{m}$, compared with $50 \mu \mathrm{m}$ accuracy for ZS. The position accuracy was estimated to be $\pm 1.7 \%$ for measuring points close to the pipe surface and $\pm 0.05 \%$ for data points taken near the centre of the pipe. Across the pipe radius, 57 data points were taken with logarithmically uniform spacing. The first point was taken with the Pitot probe touching the pipe surface, the 56th point was located at the centre of the pipe, and the 57 th point was on the other side of the pipe centre. The symmetry of the flow across the pipe, first noted by Zagarola (1996) was confirmed in the present experiment. The data were sampled using a new PC-based data acquisition system (National Instrument data acquisition boards driven by Labview software) at $500 \mathrm{~Hz}$ over a two minute period.

\section{Mean velocity results}

Figure 2 shows sample mean velocity profiles from the present work (i.e. under the new corrections) for the ZS data.

Both datasets were processed in three steps. First, the value of $\kappa$ was determined from the friction factor data by fitting

$$
\frac{1}{\sqrt{\lambda}}=C_{1} \log (\operatorname{Re} \sqrt{\lambda})+C_{2}
$$




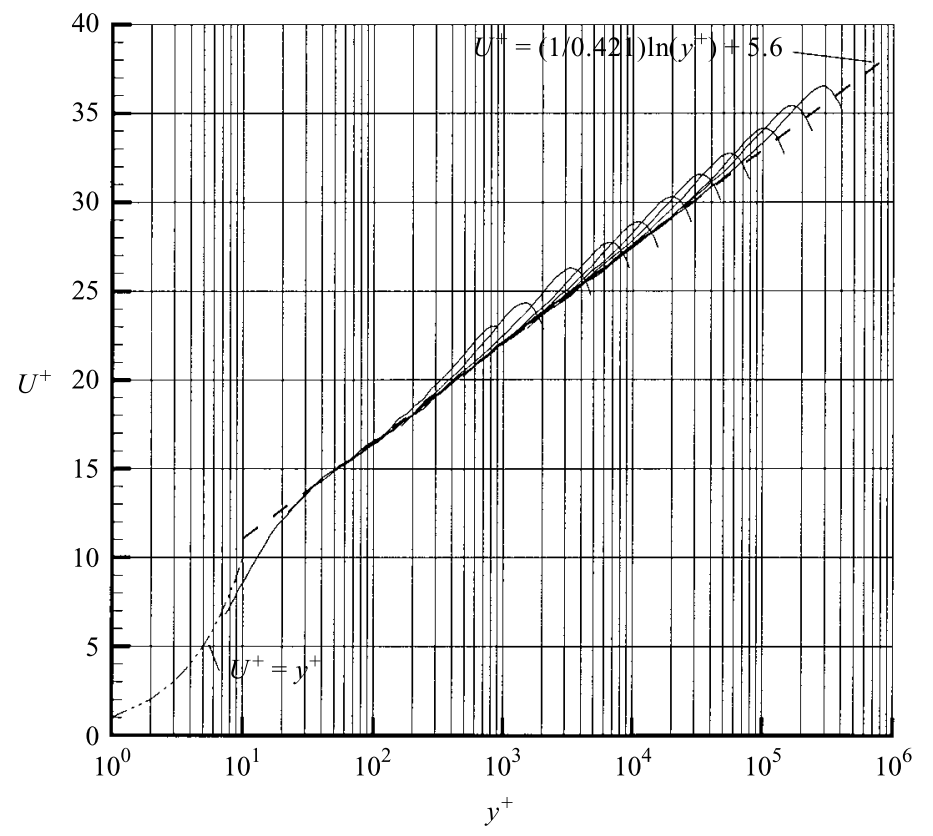

FIGURE 2. Sample mean velocity profiles for the $0.9 \mathrm{~mm}(\mathrm{ZS})$ data under the current analysis for every second Reynolds number, starting from $R e_{D}=31 \times 10^{3}$ up to $\operatorname{Re}_{D}=18 \times 10^{6}$.

to the data, where

$$
C_{1}=\frac{1}{\kappa 2 \sqrt{2} \log (e)} .
$$

Equation (2.1) is obtained by integrating the logarithmic profile from the wall to the centreline to obtain the mean velocity $\left(\bar{U}^{2}=8 u_{\tau}^{2} / \lambda\right)$. This procedure for finding $\kappa$ will hold as long as roughness is not important: the effects of roughness would cause an apparent increase in the value of $\kappa$. The curve fit was performed over different Reynolds number ranges until a range was found where $\kappa$ did not change when the range was made smaller. Finding a constant value of $\kappa$ indicates simultaneously that roughness effects are not significant, that outer similarity is observed, and that the error in deriving equation (2.1) caused by ignoring the deviation of the velocity profile from the $\log$ law close to the wall is negligible. This procedure yielded $\kappa=0.421 \pm 0.002$ (in contrast to $\kappa=0.436$ as found by ZS) when the curve fit was performed over the range $310 \times 10^{3} \leqslant R e_{D} \leqslant 13.6 \times 10^{6}$. Any range that included lower Reynolds numbers did not yield a constant value of $\kappa$. The same value of $\kappa$ over the same range of Reynolds numbers was also obtained from a curve-fit of the non-dimensional centreline velocity $U_{C L}^{+}$. This must be true for equations (1.5), (1.6) and (2.1) to hold simultaneously, and hence for a logarithmic region to exist in both inner and outer scaling.

Second, for the Pitot correction-independent data set $(y \geqslant 2 d)$, the value of the additive constant in inner scaling, $B$, was found by plotting the quantity $\psi$, where

$$
\psi=U^{+}-\frac{1}{\kappa} \ln y^{+} .
$$

In the logarithmic region, $\psi$ should be constant and equal to $B$. Figures 3 and 4 show that for $600<y^{+}<0.12 R^{+}, B$ is constant and equal to $5.60 \pm 0.08$, where the 

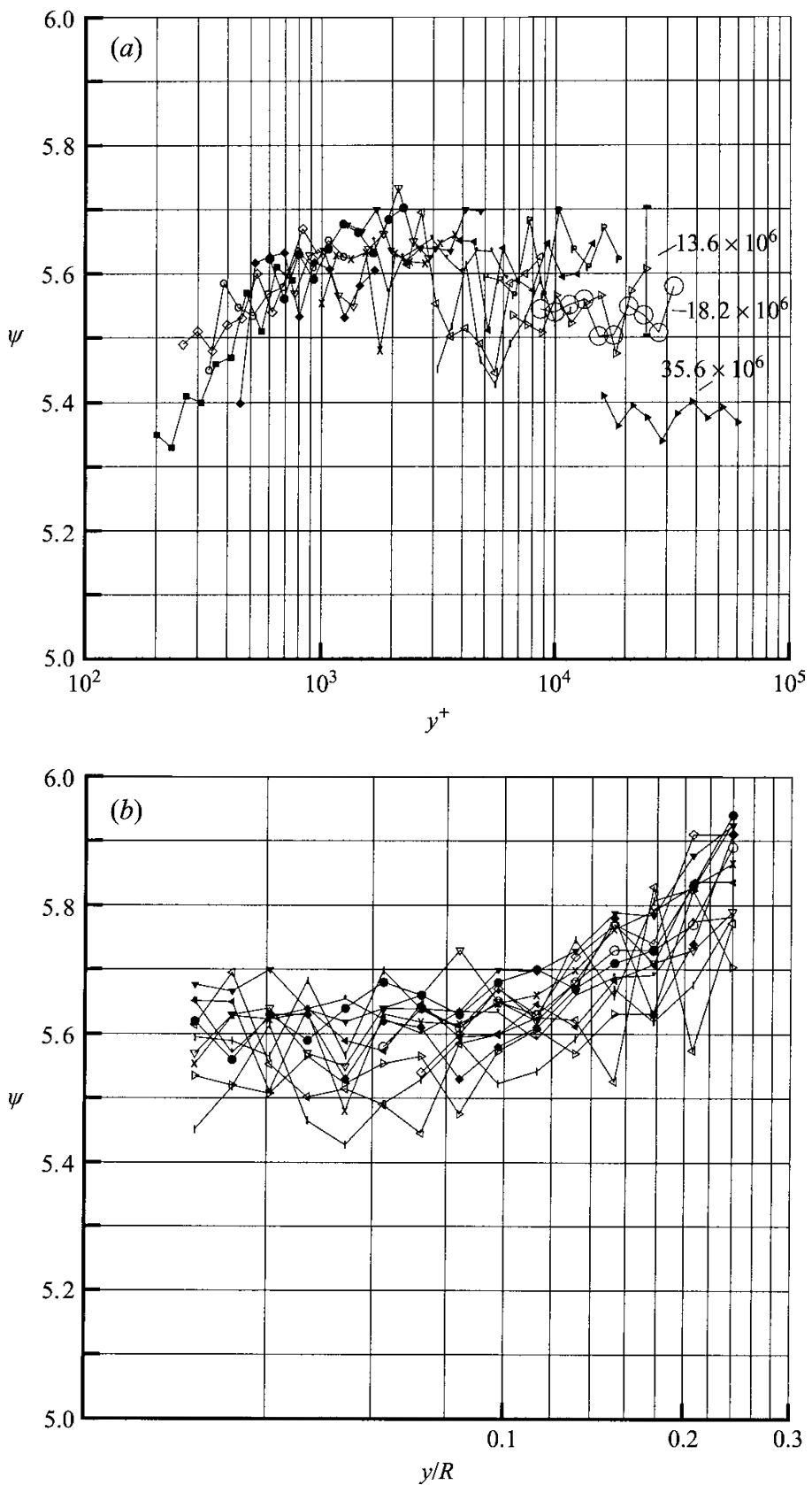

FiguRE 3. The difference between the velocity profile and the log-law for the $0.9 \mathrm{~mm}$ velocity profiles $(\kappa=0.421)$ : $(a)$ inner scaling, $y / R<0.12 ;(b)$ outer scaling, $y^{+} \geqslant 600$. Reynolds numbers $\geqslant 310 \times 10^{3}$. Symbols as in table 1 .

standard uncertainty is only 0.02 and the additional 0.06 is due to the uncertainty in $\kappa$. This compares with the value of 6.15 for $600<y^{+}<0.07 R^{+}$reported by ZS.

At the highest Reynolds numbers $\left(R e_{D} \geqslant 18 \times 10^{6}\right.$ for the $\mathrm{ZS}$ data and $R e_{D} \geqslant$ $13.6 \times 10^{6}$ for the new data), there is an apparent decrease in $B$, which may indicate the onset of roughness. However, since the decrease in $B$ from its average value 

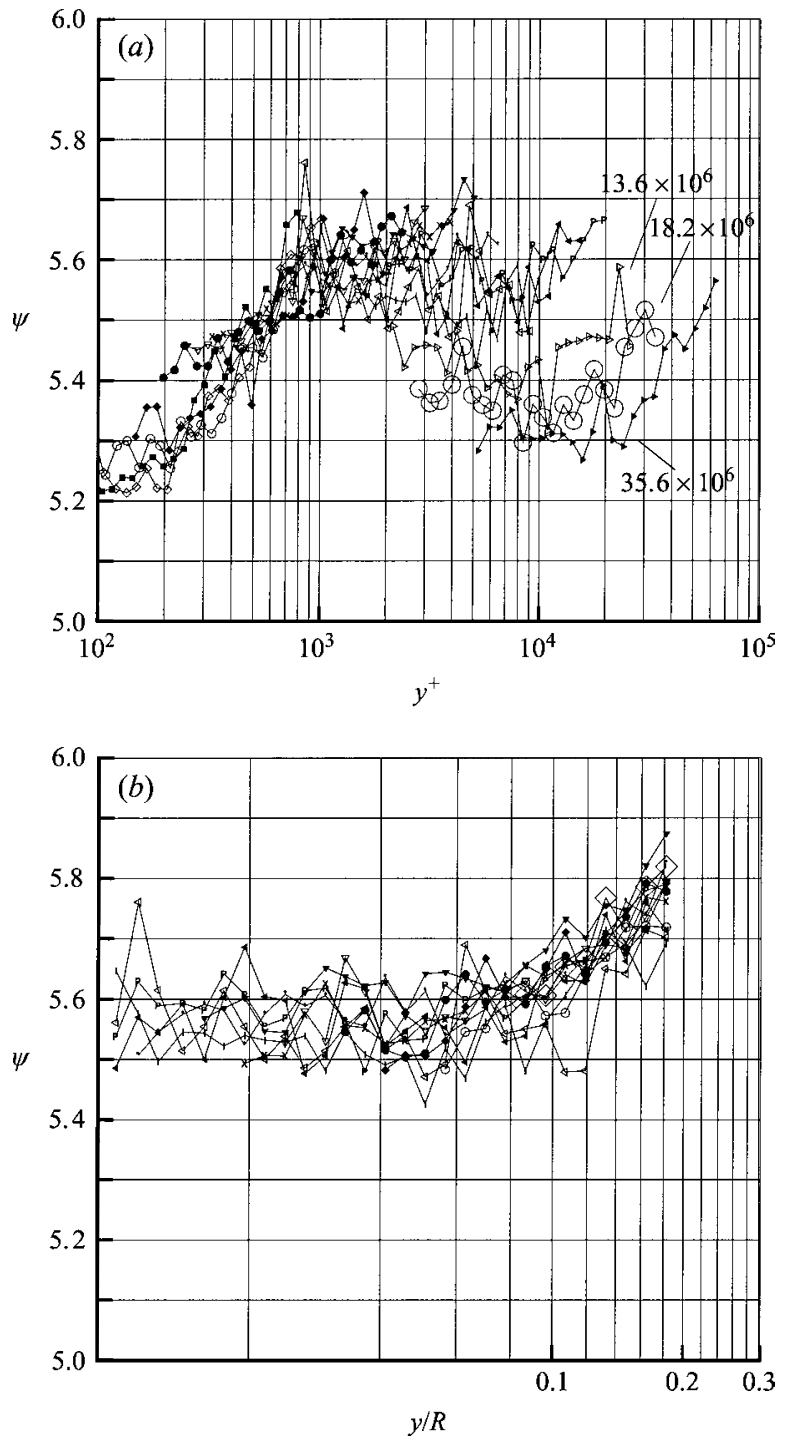

FIGURE 4 . The difference between the velocity profile and the log-law for the $0.3 \mathrm{~mm}$ velocity profiles $(\kappa=0.421)$ : $(a)$ inner scaling, $y / R<0.12 ;(b)$ outer scaling, $y^{+} \geqslant 600$. Reynolds numbers $\geqslant 310 \times 10^{3}$.

is within the uncertainty limits for most of these Reynolds numbers, no particular conclusion can be made regarding when roughness becomes important.

Third, we use the method of fractional differences defined by equation (1.8) to determine the fit of the $\log$-law with $\kappa=0.421$ and $B=5.60$ to the individual velocity profiles, over the range $600<y^{+}<0.12 R^{+}$. The experimental errors for $U$ and $U^{+}$ were estimated to be about $\pm 0.3 \%$ and $\pm 0.57 \%$, respectively. The curve-fit was judged to be unacceptable if the fractional differences $E$ were larger than the experimental uncertainty.

It may be seen from figure 5 that the new analysis presented here leads to marginally smaller fractional differences and less systematic behaviour than shown by the ZS results, which display an increasing error with increasing $y^{+}$. 

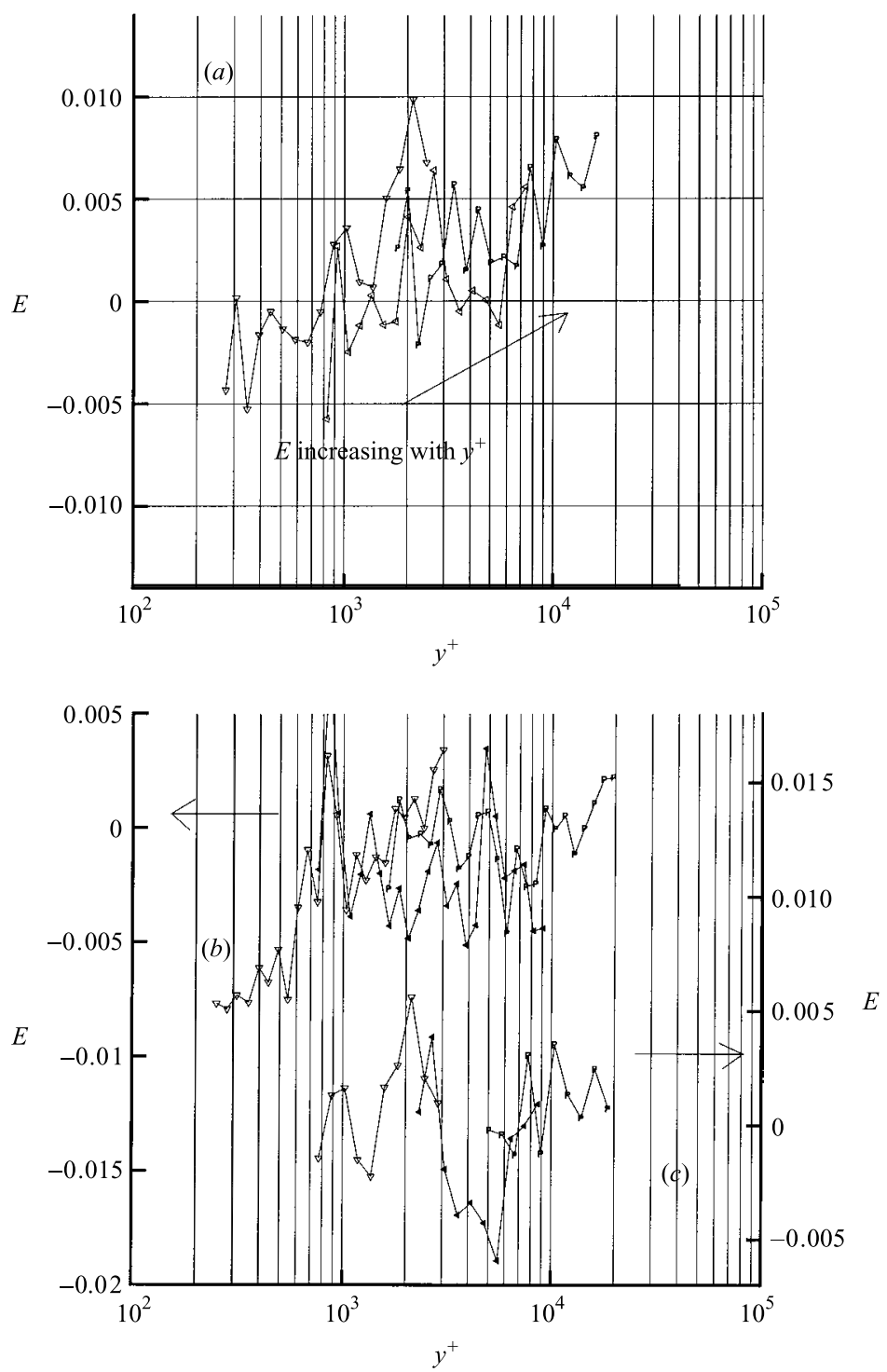

FIGURE 5. Fractional differences as defined by equation (1.8) at selected Reynolds numbers (see table 1): (a) $0.9 \mathrm{~mm}$ data, ZS log-law results; $(b) 0.3 \mathrm{~mm}$ data and $(c) 0.9 \mathrm{~mm}$ data, new log-law analysis.

For the outer-layer scaling, we now assume that $\kappa=0.421$, and calculate $B^{*}$ for each data point in the logarithmic region (that is, for $600<y^{+}<0.12 R^{+}$). Only profiles with at least six data points in the assumed logarithmic region were analysed. At the higher Reynolds numbers, 10 data points were in the assumed log region for the $0.9 \mathrm{~mm}$ probe data ( 24 points for the $0.3 \mathrm{~mm}$ probe data). The values of $B^{*}$ at each Reynolds number were averaged (no weighting) and the results are shown in figure 6. At Reynolds numbers greater than $13.6 \times 10^{6}$ the value of $B^{*}$ falls off, possibly suggesting the effects of roughness. $B^{*}$ has a constant value of $1.20 \pm 0.1$ (again the majority of the uncertainty lies in the value of $\kappa$ ) for $230 \times 10^{3} \leqslant R e_{D} \leqslant$ $13.6 \times 10^{6}$. 


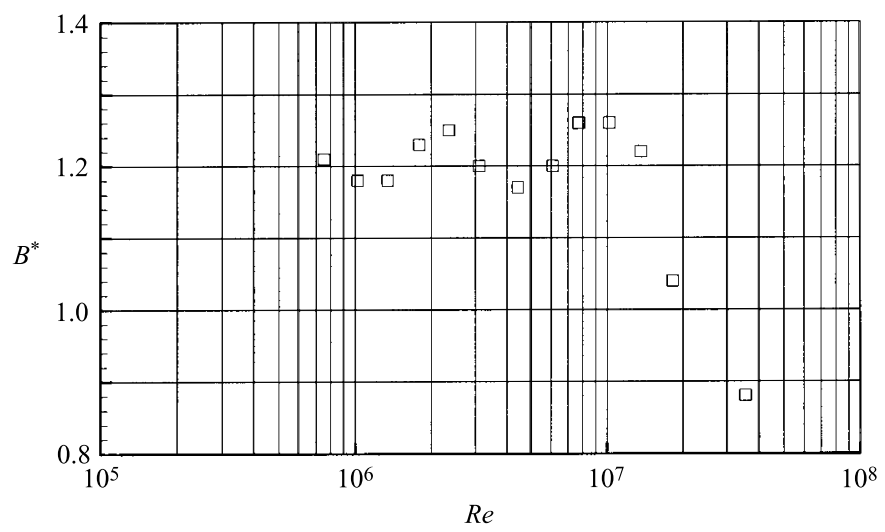

FIGURE 6. The variation of $B^{*}$ with Reynolds number: $0.9 \mathrm{~mm}$ data.

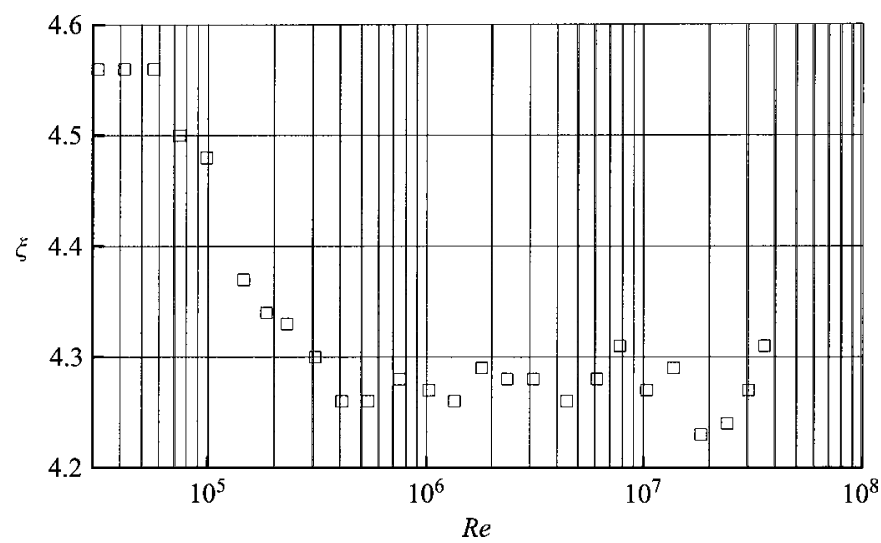

FIGURE 7. The variation of $\xi$ with Reynolds number: $0.9 \mathrm{~mm}$ data.

When $U_{C L}-\bar{U}$ is used as the velocity scale, the additive constant becomes $B^{*} / \xi$ (where $\xi=\left(U_{C L}-\bar{U}\right) / u_{\tau} \approx 4.28$, see below) which has a value of $0.280 \pm 0.02$, and the slope becomes $1 / \kappa \xi$ which has a value of $1 /(1.798 \pm 0.01)$. For $600 / R^{+}<y / R<0.12$, a logarithmic overlap region appears to exist which can be accurately represented by

$$
\frac{U_{C L}-U}{U_{C L}-\bar{U}}=\frac{-1}{1.798} \ln \eta+0.28
$$

or

$$
\frac{U_{C L}-U}{u_{\tau}}=\frac{-1}{0.421} \ln \eta+1.20 .
$$

The velocity profile data are within $\pm 1.0 \%$ (95\% confidence interval) of equations (2.4) and (2.5). The relatively large uncertainty can be attributed to the uncertainty in $U_{C L}-U$, which is the difference between two large numbers.

As indicated earlier, there was no region of Reynolds number less than $R e_{D}=310 \times 10^{3}$ for which the friction factor data or centreline velocity $U_{C L}^{+}$yielded a constant value of $\kappa$. (Note that at $\operatorname{Re}_{D}=230 \times 10^{3}, y^{+}=600$ at $y / R \approx 0.12$ and hence the region of the predicted $\log$ law would be negligible.) As noted above, the ratio of the velocity scales $\xi=\left(U_{C L}-\bar{U}\right) / u_{\tau}$ must be constant for there to exist a log law in both inner and outer scaling in the accepted forms. It can be seen from figure 7 


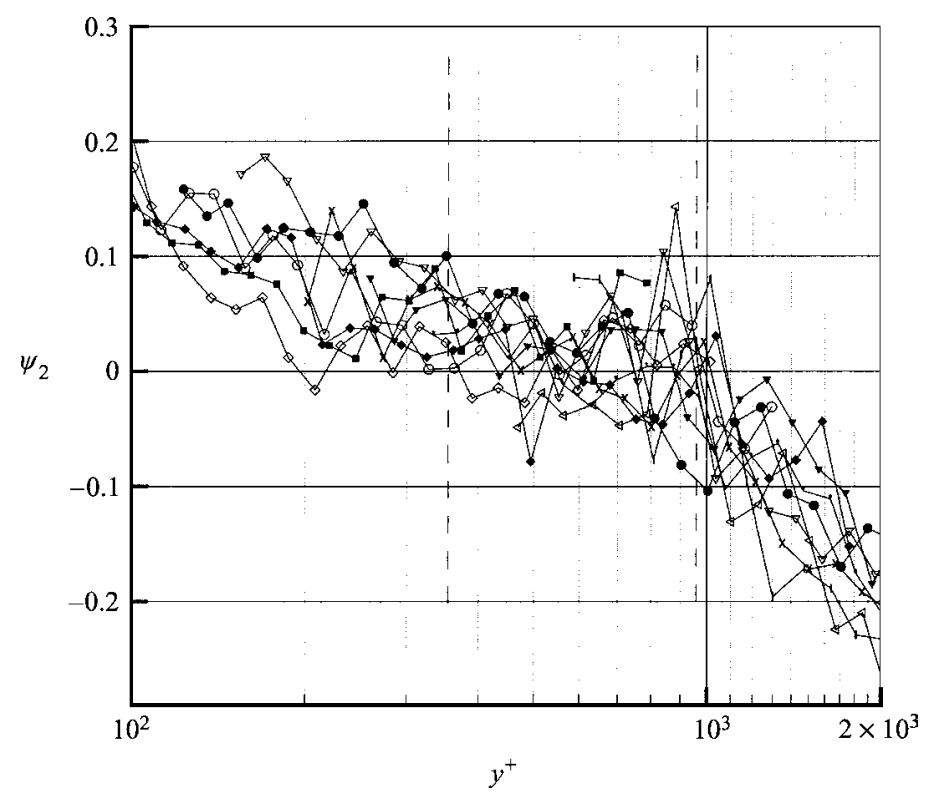

FIGURE 8 . The difference between the velocity profiles and a logarithmic region with slope 1/0.385 $\left(y^{+}=350,950\right.$ marked by dashed lines $): 0.3 \mathrm{~mm}$ data.

that this ratio of velocity scales reaches a constant value of approximately 4.28 for $R e_{D} \geqslant 310 \times 10^{3}$, which agrees well with the limits on where a log law was observed in the data.

Up to this point, only data points for $y \geqslant 2 d$ were included in the analysis, and the data were therefore independent of Pitot tube corrections. However, to study the near-wall region we need additional points near the wall, and Pitot tube corrections become important. We will use the correction suggested by McKeon et al. (2003), on the basis that it produces good agreement among different diameter Pitot data sets down to $y \approx d$, considerably better than other methods such as MacMillan (1956) and Chue (1975). However, as indicated earlier, all these corrections lead to the same conclusions when data for $y \geqslant 2 d$ are analysed.

It was found that log-laws with the constants determined by Österlund et al. (2000) $(\kappa=0.38$ and $B=4.1)$ and Zanoun et al. (2002) $(\kappa=0.379$ and $B=4.05)$ fit our pipe flow measurements quite well, but only for the small region where $350<y^{+}<950$, compared with their range of $200 / \delta^{+}<y / \delta<0.15$. The fit could be improved a little by using the values 0.385 and 4.15 , as shown in figure 8 , where the difference $\psi_{2}$ is defined as

$$
\psi_{2}=U^{+}-\frac{1}{0.385} \ln y^{+}-4.15 .
$$

However, even if this region scales logarithmically, similarity is not complete since the ratio $\left(U_{C L}-\bar{U}\right) / u_{\tau}$ is not constant in this region. Furthermore, the friction factor data for this range of Reynolds numbers does not lead to a constant value of $\kappa$, so that this result may simply be spurious.

Finally, a power law is found to be a good fit for the region $50<y^{+}<300$ or $0.12 R^{+}$, where the constants were found using a least-squares determination to be given by $C=8.47$ and $\gamma=0.142$. The variation of $\psi_{3}$ and $E$ is shown in figure 9 

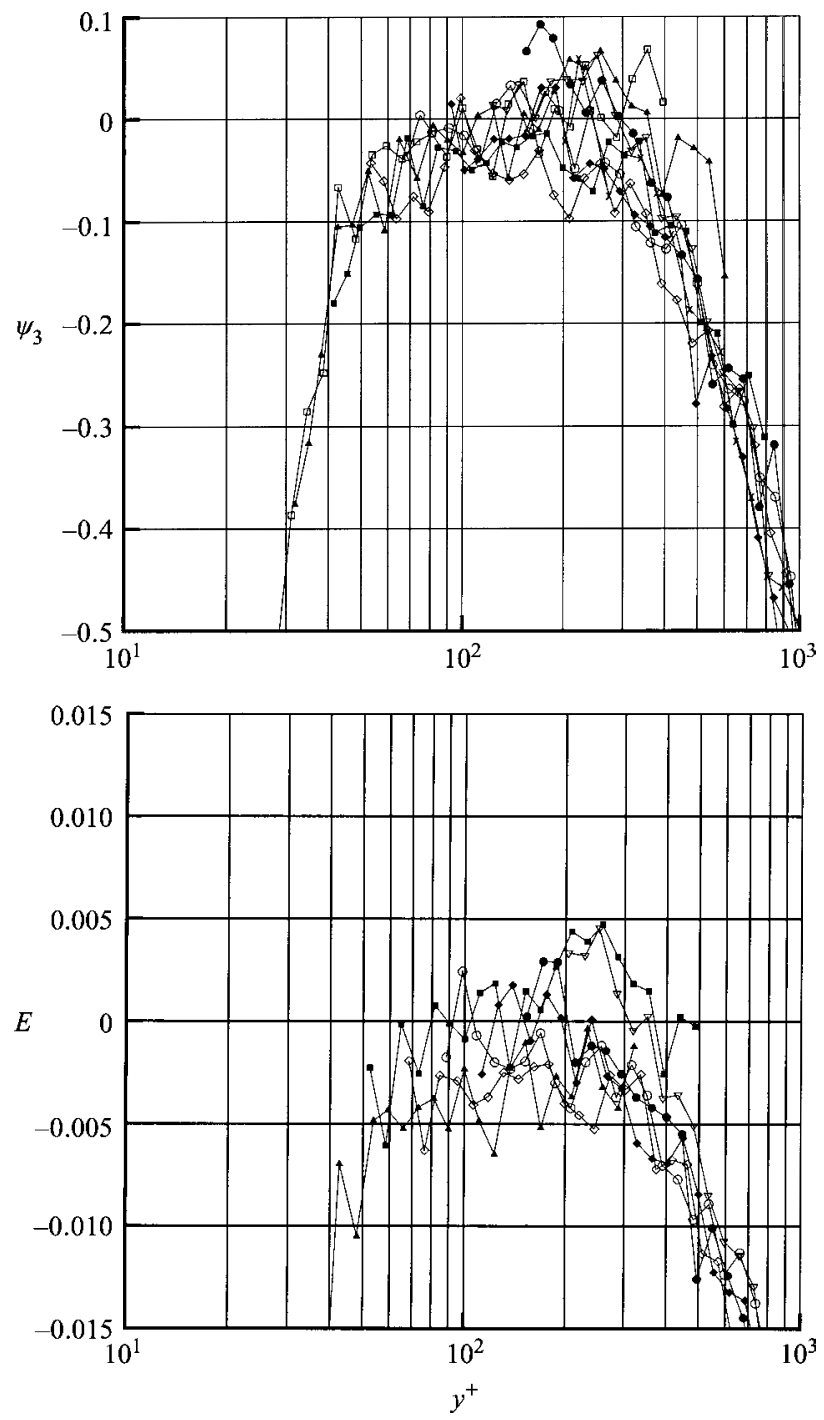

FiguRE 9. The difference, $\psi_{3}$, and fractional difference, $E$, between the velocity profiles and a power law $(C=8.47$ and $\lambda=0.142): 0.3 \mathrm{~mm}$ data.

where

$$
\psi_{3}=U^{+}-8.47 y^{+0.142} .
$$

The $U^{+}$data are within $\pm 0.33 \%$ (95\% confidence limits) of this power law over this range, which is close to the experimental uncertainty in $U^{+}( \pm 0.57 \%)$. Note that this region is similar to that found by $\mathrm{ZS}$, who reported a power law with $C=8.70$ and $\lambda=0.137$ for the region $60<y^{+}<500$ or $0.15 R^{+}$.

\section{Conclusions and discussion}

Use of the appropriate high-Reynolds-number static pressure correction leads to significant changes to the analysis of ZS. The new results confirm the presence of a power-law region near the wall and, for Reynolds numbers greater than $200 \times 10^{3}$, 
a logarithmic region further out, but the limits of these regions and some of the constants are different. In particular, the power law is found only for $50<y^{+}<300$ (instead of $60<y^{+}<500$ as reported by ZS), and the multiplicative constant and exponent are 8.48 and 0.142 , respectively (instead of 8.70 and 0.137 ).

The log law is found for $600<y^{+}<0.12 R^{+}$(instead of $600<y^{+}<0.07 R^{+}$), and the von Kármán constant $\kappa$, the additive constant $B$ for the log law using inner flow scaling, and the additive constant $B^{*}$ for the log law using outer scaling are found to be $0.421 \pm 0.002,5.60 \pm 0.08$ and $1.20 \pm 0.1$, respectively, with $95 \%$ confidence level (compared with $0.436 \pm 0.002,6.15 \pm 0.08$, and $1.51 \pm 0.03$ found by ZS). In addition, the new data confirm the conclusions by ZS that their pipe flow data are not affected by surface roughness until the highest Reynolds numbers $\left(\operatorname{Re}_{D}>13.6 \times 10^{6}\right.$, at a minimum).

In the region $350<y^{+}<950$, the data could be said to be in reasonably good agreement with the findings by Österlund et al., who suggested a log law with $\kappa=0.38$ and $B=4.1$ for $200<y^{+}<0.15 R^{+}$for turbulent boundary layers. The values of $\kappa$ and $B$ reported by Österlund et al. are markedly different, however, from the values given here for the region of complete similarity which occurs further from the wall, that is for $600<y^{+}<0.12 R^{+}$, and the power and log law scalings given in this paper represent the data more accurately.

The support of ONR under Grant Nos. N00014-98-1-0525, N00014-99-1-0340, and N00014-03-1-0320 is gratefully acknowledged. J.F.M. is indebted to the Engineering and Physical Sciences Research Council (grants GR/M64536/01 and GR/R48193/01), the Royal Academy of Engineering (England) and the Leverhulme Trust (grant $\mathrm{F} / 07058 / \mathrm{H}$ ) for financial support.

\section{REFERENCES}

Bradshaw, P. 1976 Topics in Applied Physics, Turbulence. Springer.

Chue, S. H. 1975 Pressure probes for fluid measurement. Prog Aerospace Sci. 16 (2), 147-223.

MacMillan, F. A. 1956 Experiments on Pitot-tubes in shear flow. Ministry of Supply, Aero. Res. Counc. R \& M 3028.

McKeon, B. J., Li, J., Jiang, W., Morrison, J. F. \& Smits, A. J. 2003 Pitot probe corrections in fully-developed turbulent pipe flow. Meas. Sci. Tech. 14, 1449-1458.

McKeon, B. J. \& Smits, A. J. 2002 Static pressure correction in high Reynolds number fullydeveloped turbulent pipe flow. Meas. Sci. Tech. 13, 1608-1614.

Millikan, C. B. 1938 A critical discussion of turbulent flows in channels and circular tubes. Proc. Fifth Intl Cong. Appl. Mech., pp. 386-392.

Österlund, J. M., Johansson, A. V., Nagib, H. M. \& Hites, M. H. 2000 A note on the overlap region in turbulent boundary layers. Phys. Fluids 12, 1-4.

Perry, A. E., Hafez, S. \& Chong, M. S. 2001 A possible reinterpretation of the Princeton Superpipe data. J. Fluid Mech. 439, 395-401.

Schlichting, H. 1979 Boundary Layer Theory. McGraw-Hill.

SHAw, R. 1960 The influence of hole dimensions on static pressure measurements. J. Fluid Mech. 7, $550-564$.

Zagarola, M. V. 1996 Mean flow scaling in turbulent pipe flow. PhD thesis, Princeton University.

Zagarola, M. V., Perry A. E. \& Smits, A. J. 1997 Log laws or power laws: The scaling in the overlap region. Phys. Fluids 9, 2094-2100.

Zagarola, M. V. \& Smits, A. J. 1998 Mean flow scaling in turbulent pipe flow. J. Fluid Mech. 373, 33-79 (referred to herein as ZS).

Zanoun, E.-S., Nagib, H., Durst, F. \& Monkewitz, P. 2002 Higher Reynolds number channel data and their comparison to recent asymptotic theory (Invited). AIAA Paper 2002-1102, A02-14297. 